\title{
AVALIAÇÃO DOS RESULTADOS DO TRATAMENTO DE 14 DOENTES DE CARCINOMA ESPINOCELULAR ANAL
}

\author{
LUÍS AUGUSTO PALMA DALLAN ${ }^{1}$, SYLVIA HELOISA ARANTES CRUZ ${ }^{1}$, DALTRO LEMOS DA ROSA ${ }^{1}$, FANG \\ CHIA BIN ${ }^{1}$, SIDNEY ROBERTO NADAL ${ }^{1}$, PERETZ CAPELHUCHNIK ${ }^{1}$, WILMAR ARTUR KLUG ${ }^{1}$
}

${ }^{1}$ Departamento de Cirurgia da Faculdade de Ciências Médicas da Santa Casa de São Paulo, São Paulo, Brasil

DALLAN LAP, CRUZ SHA, ROSA DL,BIN FC, NADAL SR, CAPELHUCHNIK P, KLUG WA. Avaliação dos Resultados do Tratamento de 14 Doentes de Carcinoma Espinocelular Anal. Rev bras Coloproct, 2005;26(1):34-40.

RESUMO: A radioquimioterapia (RT/QT) tornou-se o tratamento de escolha para o carcinoma espinocelular anal (CEC). Na recidiva local ou na persistência da doença, deve-se instituir o tratamento cirúrgico.

Objetivo: O objetivo deste estudo retrospectivo foi analisar os resultados do tratamento de doentes de CEC anal.

Método: Acompanhamos 17 pacientes com diagnóstico anátomo-patológico de carcinoma espinocelular anal. Eram 14 (82,3\%) do sexo feminino e três $(\mathbf{1 7 , 8 \%})$ do masculino. A idade variou de 36 a 78 anos, com média de idade de 59,1 anos. Utilizando a classificação TNM, tivemos quatro $(23,6 \%)$ no estádio I, seis $(35,2 \%)$ no II, quatro $(23,6 \%)$ no IIIa e três $(17,6 \%)$ no IIIb. Todos foram submetidos a tratamento inicial com RT/QT, exceto um submetido a ressecção local. Definimos que a biópsia negativa, realizada entre 12 e 16 semanas após esse tratamento, determinaria o controle local da doença.

Resultados: Perdemos seguimento de três doentes (17,6\%). Seguimos os 14 restantes $(82,3 \%)$ entre um e cinco anos. Todos os doentes nos estádios I e II (10) apresentaram regressão total da doença, enquanto que três (75\%) nos estádios IIIa e IIIb tiveram remissão completa. Realizamos a amputação abdomino-perineal de resgate em dois doentes e ressecção local em outros dois. A recidiva local ocorreu em dois (20\%) nos estádios I e II e em dois (75\%) nos estádios mais avançados (IIIa e IIIb). A sobrevivência em 3 anos foi de $100 \%$ nos que se encontravam nos estádios I e II, embora o controle da doença fosse atingido em oito (80\%). Nos quatro doentes que estavam nos estádios IIIa e IIIb, a sobrevivência em um ano foi de $75 \%$ e em três anos foi de $25 \%$. Esse último permanece livre da doença. Complicações do tratamento radioterápico ocorreram em oito doentes $(57,1 \%)$. Nenhum óbito foi constatado durante o tratamento RT/QT. Os dois doentes, $(14,3 \%)$ com sorologia positiva para HIV, apresentavam infecção anal pelo Papilomavírus humano (HPV).

Conclusão: A análise dos nossos resultados evidenciou que o esquema de tratamento empregado foi efetivo para o controle local e preservação da função esfincteriana do ânus e que, na falha do tratamento radioquimioterápico, a operação de resgate controlou localmente a doença.

Descritores: Carcinoma de células escamosas, Radioterapia, Quimioterapia.

\section{INTRODUÇÃO}

A incidência do carcinoma anal corresponde a $1,5 \%$ dos tumores do aparelho digestivo. ${ }^{1,2}$ Entretanto, com o surgimento da AIDS, esse tipo de tumor tem apresentado freqüência 25 a 50 vezes maior nos infectados pelo HIV, principalmente homens entre 30 e 40 anos, modificando a epidemiologia dessa neoplasia. ${ }^{2-4}$ Esse aumento vem sendo observado desde os anos 80 , especialmente entre praticantes de sexo anal receptivo, ${ }^{5} \mathrm{e}$ parece estar intimamente relacionado com a infecção pelo Papilomavirus humano (HPV). ${ }^{6,7}$ Além

Trabalho realizado na Disciplina de Coloproctologia do Departamento de Cirurgia da Faculdade de Ciências Médicas da Santa Casa de São Paulo.

$\overline{\text { Recebido em 23/02/2006 }}$

Aceito para publicação em 10/03/2006 
disso, acredita-se que o aumento do tempo de expectativa de vida dos doentes HIV-positivos, proporcionado pelo atual esquema de drogas antirretrovirais, possa predispor o aparecimento de maior número de tumores. ${ }^{8,9,10}$

Os HPVs dos tipos 5, 8, 16, 18, 31 e 45 são os mais encontrados nas lesões neoplásicas e são chamados de alto risco. ${ }^{11,12}$ A ligação de proteína E6 dos HPVs de alto risco à proteína P53 causa rápida degradação dessa última, levando à perturbação do controle do crescimento celular. ${ }^{13}$ Acredita-se que o potencial oncogênico desses vírus ocorra, em parte, devido a essa interação. ${ }^{14,15}$

São denominados como carcinoma espinocelular (CEC), quando surgem no epitélio pavimentoso estratificado do canal ou da margem anal, e como carcinoma cloacogênico (ou basalóide, ou transicional), tipo mais raro, correspondendo a $30 \%$ das lesões, caso se desenvolva no epitélio de transição da linha pectínea. ${ }^{16}$

A maioria dos doentes refere saliência ou caroço no ânus, associado a prurido, dor e/ou desconforto anal, podendo apresentar hematoquezia ou sangramento de pequeno volume às evacuações, percebido no vaso sanitário, no papel ou nas roupas íntimas. ${ }^{17}$ Nódulos inguinais podem ser notados, sobretudo nos casos mais avançados. ${ }^{17}$

A anamnese e o exame proctológico levam à suspeita diagnóstica que será confirmada pela avaliação anátomo-patológica. Para conhecer o estádio da doença, serão necessários exames para avaliar a profundidade da lesão e a invasão do aparelho esfincteriano e de outras estruturas vizinhas ${ }^{17,18}$ e o comprometimento linfonodal local, inguinal e mais distante. ${ }^{17}$ Para tanto, solicitamos exames de imagem como ultrassonografia endoanal, tomografia computadorizada ou ressonância nuclear magnética. ${ }^{19}$ As metástases ocorrem entre 10 e $15 \%$ dos casos, sendo o fígado e os pulmões os órgãos mais atingidos, o que constitui motivo para sua avaliação. ${ }^{20}$

O tratamento desse tumor atravessou profunda transformação nos últimos 20 anos. Antes desse período, preconizava-se a amputação abdominoperineal (AAP), que necessita de colostomia definitiva. ${ }^{21,22}$ Atualmente, elevado índice de resposta com a associação da quimioterapia com a radioterapia (RT/QT), fez desse o método de escolha para tratar o CEC anal. ${ }^{21,23}$ Mesmo a RT/QT, que tem grande aceitação desde os estudos de Nigro et al, ${ }^{24}$ usando 5fluorouracil e mitomicina $\mathrm{C}$, associados com 20 sessões de 45 a 55 Gy, vem passando por modificações no intuito de definir o esquema terapêutico que apresente os melhores índices de cura associados com a menor toxicidade. ${ }^{22,25,26}$ A remissão dos tumores ocorre entre 80 e $90 \%$, porém com bons resultados entre 50 e $75 \%$ nas lesões maiores que $5 \mathrm{~cm} .{ }^{23}$ Todavia, o esquema terapêutico depende da localização do tumor. Quando localizado no canal anal, a RT/QT está inicialmente indicada. Caso o tumor seja da margem anal, a ressecção cirúrgica é feita antes, exceto nas lesões muito volumosas, nas quais a RT/QT será utilizada previamente. ${ }^{27}$

O tratamento cirúrgico pode cursar com lesões dos nervos pélvicos, acarretando danos ao aparelho reprodutor e urinário, e perda da função esfincteriana, impondo a realização de colostomia. ${ }^{20-23}$ A preservação da função esfincteriana é uma das vantagens da RT/ QT sobre o tratamento cirúrgico, ${ }^{17}$ sendo obtida em 80 a $100 \%$ dos casos. ${ }^{26,28}$ Entretanto, a RT/QT não é isenta de desvantagens. Apresenta efeitos colaterais tóxicos, locais e sistêmicos, que incluem mielossupressão, dificuldade de cicatrização, alterações da função esfincteriana, dermatite, fístula e mucosite, que são mais intensos nos doentes imunodeprimidos. ${ }^{29}$

$\mathrm{O}$ atual esquema, proposto para tratamento dos CEC anais, é considerado efetivo e apresenta sobrevivência de 5 anos entre 68 e $86 \%^{19,21,25,26}$ e de 10 anos de $75 \% .{ }^{28}$ As recidivas ocorrem entre 8 e $21 \%$, porém são passíveis de cura com procedimentos operatórios. ${ }^{19,21,26}$ Entretanto, o estádio da doença é fator prognóstico para aumento da sobrevivência. ${ }^{26}$ Os exames complementares são fundamentais no seguimento pós-tratamento, para diagnóstico das recidivas locorregionais ou metástases. ${ }^{23,25-29} \mathrm{Na}$ recidiva local ou na persistência da doença após RT/ QT, devemos instituir as operações de resgate. Conforme o estádio do tumor podemos realizar tanto a ressecção local como a AAP. ${ }^{20-23} \mathrm{O}$ tratamento cirúrgico deve ser individualizado e adaptado às condições clínicas do doente. ${ }^{27}$ Além disso, na presença de metástases, as operações podem ter apenas intuito paliativo, aliviando a dor, estenose ou sangramento. ${ }^{27}$ $\mathrm{O}$ objetivo deste estudo retrospectivo foi analisar os resultados do tratamento dos nossos doentes de CEC anal. 


\section{METODO}

Entre janeiro de 1999 e dezembro de 2004, atendemos 17 doentes de CEC anal. A idade variou de 36 a 78 anos, com média de 59,1 anos, sendo 14 $(82,3 \%)$ do sexo feminino e três $(17,8 \%)$ do masculino, com relação de 4,6:1.

A classificação TNM de 1997, adotada como base para a avaliação dos dados, revelou que quatro doentes $(23,6 \%)$ encontravam-se no estádio I, seis no II, quatro no IIIa e três no IIIb, conforme consta na Tabela-1.

O tratamento inicial consistiu em RT/QT, conforme o protocolo de Nigro modificado. Todos receberam 5-fluorouracil (5-FU) associado com mitomicina-C (MTC) ou cisplatina (CIS). A RT foi administrada em campo pélvico com dose total média de 45 Gy, variando de 35 a 50 Gy, com duração entre quatro e oito semanas. Nos doentes com comprometimento linfonodal inguinal foram aplicadas doses de reforço radioterápico de 10 Gy na região. Após 12 a 16 semanas do término do esquema RT/QT, realizamos biópsia para avaliar a eficácia da terapia.

Operamos aqueles sem regressão completa ou com recidivas. A técnica usada dependeu do estádio da lesão. Nos estádios I e II fizemos a ressecção local, ao passo que nos estádios IIIa e IIIb indicamos a AAP e radioterapia inguinal adjuvante, na dose de $10 \mathrm{~Gy}$. Na presença de metástases, indicamos QT adjuvante com 5-FU e CIS. Um dos doentes, submetido a ressecção de plicoma anal e cujo exame anatomopatológico revelou CEC, recebeu RT/QT.

O seguimento ambulatorial foi trimestral durante os dois primeiros anos, semestral nos três anos

Tabela 1 - Classificação TNM dos doentes de CEC anal no Hospital Central da Faculdade de Ciências Médicas da Santa Casa de São Paulo. 2005

Número de doentes Porcentagem (\%)

\begin{tabular}{lcc}
\hline Estádio I & 4 & 23,6 \\
Estádio II & 6 & 35,2 \\
Estádio IIIa & 4 & 23,6 \\
Estádio IIIb & 3 & 17,6 \\
Total & 17 & 100,0 \\
\hline
\end{tabular}

Fonte: SAME Sta. Casa de São Paulo. subsequientes, e anual após o quinto ano, no intuito de verificar a recidiva tumoral ou a presença de metástases. Os exames consistiram de biópsias locais, radiografia torácica, ultrassonografia abdominal e tomografia computadorizada. Após dois anos, os exames foram realizados anualmente.

\section{RESULTADOS}

Perdemos o seguimento de três doentes. Os 14 restantes têm seguimento entre um e cinco anos, com média de 37 meses. Desses, 13 (92,8\%) apresentaram regressão completa do câncer anal., dois com ressecção local e 11 após RT/QT $(83,3 \%)$ Os 10 doentes nos estádios I e II e três dos quatro (75\%) nos estádios IIIa e IIIb tiveram remissão completa. (Tabela-2).

A recidiva local ocorreu em quatro $(30,8 \%)$ dos 13 doentes com remissão da doença, sendo dois (20\%) nos estádios I e II e outros dois $(66,7 \%)$ nos estádios IIIa e IIIb. (Tabela-3).

Tabela 2 - Distribuição de 14 doentes de CEC anal conforme a remissão com o tratamento e o estádio da doença. 2005

\begin{tabular}{ccc}
\hline estádio & Controlados/Total & Porcentagem (\%) \\
\hline I e II & $10 / 10$ & 100 \\
IIIa e IIIb & $03 / 04$ & 75 \\
Total & $13 / 14$ & 92,8 \\
\hline
\end{tabular}

Fonte: SAME Sta. Casa de São Paulo.

Tabela 3 - Distribuição de 13 doentes de CEC anal com remissão após tratamento conforme a ocorrência de recidiva local e o estádio da doença. 2005

\begin{tabular}{ccc}
\hline estádio & Recidivados/Total & Porcentagem (\%) \\
\hline I e II & $02 / 10$ & 20 \\
IIIa e IIIb & $02 / 03$ & 66,7 \\
Total & $04 / 13$ & 30,8 \\
\hline
\end{tabular}

Teste exato de Fisher $p=0,203$ (não significante)

Fonte: SAME Sta. Casa de São Paulo. 
Observamos sobrevivência em três anos em 11 doentes $(78,5 \%)$, sendo 10 nos estádios Ie II, embora dois apresentassem recidiva. Dos quatro doentes nos estádios IIIa e IIIb, três doentes sobreviveram 12 meses e só um permanecia vivo e livre de doença após o terceiro ano (Tabela-4). A análise estatística revelou que os doentes nos estádios mais avançados tiveram sobrevivência de três anos, inferior àqueles dos estádios mais iniciais $(\mathrm{p}=0,011)$.

O paciente inicialmente tratado com ressecção local de plicoma com CEC, e RT/QT posterior, evoluiu com remissão completa e encontra-se livre da doença. Realizamos operação de resgate em quatro doentes $(28,6 \%)$, sendo a ressecção local nos dois doentes que apresentaram recidivas nos estádios I e II, e a AAP de resgate associada à RT inguinal adjuvante nos dois dos estádios IIIa e IIIb e com recidiva. Noutro no estádio IIIb, que teve indicação de AAP, constatamos carcinomatose, a lesão foi considerada irressecável e a morte ocorreu após três meses.

Os dois pacientes que submetemos a ressecção local encontram-se vivos, um deles livre da doença, com seguimento de 40 meses. Entretanto, o outro apresentou recidiva após 7 meses, que foi ressecada, permanecendo livre de doença após 12 meses. Dos dois tratados com AAP, um morreu após 12 meses e o outro está livre da doença após 36 meses. A taxa de sobrevivência global em três anos após operação de resgate foi de $75 \%$. (Tabela-5).

Complicações tardias do tratamento radioterápico ocorreram em oito doentes $(57,1 \%)$. Foram elas: dermatite $(42,8 \%)$, retite e colite actínica $(50 \%)$, estenose retal $(21,4 \%)$, incontinência anal $(14,3 \%)$ e fístula reto-vaginal $(7,1 \%)$. Apenas dois doentes $(14,3 \%)$ evoluíram com incontinência anal, portanto obtivemos índice de preservação da função esfincteriana de $84,7 \%$.
Tabela 5 - Distribuição de quatro doentes de CEC anal após cirurgia de resgate conforme a sobrevivência em três anos e o estádio da doença. 2005

\begin{tabular}{ccc}
\hline estádio & Vivos/Total & Porcentagem (\%) \\
\hline I e II & $02 / 02$ & 100 \\
IIIa e IIIb & $01 / 02$ & 50 \\
Total & $03 / 04$ & 75 \\
\hline
\end{tabular}

Fonte: SAME Sta. Casa de São Paulo.

Observamos a concomitância da infecção anal pelo HPV em 2 doentes (14,3\%), um deles no estádio IIIa e o outro no IIIb. Ambos eram portadores da AIDS em tratamento com antirretrovirais. Perdemos o seguimento de um e o outro permanece vivo após três anos, embora com recidiva da doença.

\section{DISCUSSÃO}

O CEC anal predominava no sexo feminino na proporção de 5:1, e na faixa etária entre a sexta e sétima décadas da vida. ${ }^{3}$ Entretanto, entre os portadores da infecção pelo HIV, a doença vem ocorrendo em doentes mais jovens e no sexo masculino. ${ }^{2-4} \mathrm{Na}$ nossa casuística, observamos média etária de 59 anos e 4,6 mulheres para cada homem, todavia, atendemos apenas dois portadores do HIV, ambos homens e mais jovens.

Constatamos infecção pelo HIV em dois doentes. Foram tratados da mesma maneira que os não infectados e apresentaram resultados semelhantes, conforme citado na literatura. ${ }^{30,31}$ A história pregressa de condilomas acuminados anais foi citada por ambos. Entre os doentes soronegativos para o HIV, a associação

Tabela 4 - Distribuição de 14 doentes de CEC anal conforme a sobrevivência em um e três anos e o estádio da doença. 2005

\begin{tabular}{ccc}
\hline estádio & um ano de sobrevivência & três anos de sobrevivência \\
\hline I e II & $10 / 10(100 \%)$ & $10 / 10(100 \%)$ \\
IIIa e IIIb & $03 / 04(75 \%)$ & $01 / 04(25, \%)(*)$ \\
Total & $13 / 14(92,8 \%)$ & $11 / 14(78,5 \%)$ \\
\hline
\end{tabular}

* Teste exato de Fisher $p=0,011$ (significante)

Fonte: SAME Sta. Casa de São Paulo. 
com formas clínicas da infecção pelo HPV não ocorreu. Tampouco pesquisamos o DNA viral nas células tumorais. Desta forma não sabemos em quantos dos HIV-negativos havia a presença do HPV.

É necessário conhecer a localização do tumor para indicar a terapia mais correta. ${ }^{27}$ Embora a RT/ QT seja o esquema terapêutico de escolha, nos tumores pequenos da borda anal a ressecção pode ser realizada, a princípio. ${ }^{27,32}$ Dentre nossos 14 doentes com seguimento, dois tinham tumores com origem na margem e 12 no canal anal. Em um dos portadores da lesão da borda anal, o achado foi incidental, após ressecção de plicoma. A literatura refere que os tumores originados no canal anal são mais comuns, numa relação de seis para um com os da margem anal. $^{32}$

Avaliando nossos resultados observamos que a RT/QT empregada nas lesões do canal anal foi efetiva no controle local do tumor em $83,3 \%$ dos casos. São dados semelhantes aos da literatura que mostraram eficácia entre 50 e $90 \%$ dos doentes, dependendo do tamanho do tumor ${ }^{23}$ e do estádio da doença. ${ }^{33}$ Também foi citado que a RT/QT é menos eficaz nos doentes mais idosos. ${ }^{33}$ Conseguimos a remissão completa em todos os tumores nos estádios mais iniciais (I e II) e em 75\% daqueles nos estádios IIIa e IIIb. Já entre os tumores da margem anal o controle cirúrgico foi obtido nos dois doentes, sendo que a RT/QT foi realizada posteriormente.

Estatísticas revelaram incidência de $60 \%$ e $17 \%$ de linfonodos comprometidos; $75 \%$ e $6 \%$ de recidivas; $62 \%$ e $85 \%$ de respostas à radio e quimioterapia; $80 \%$ e $30 \%$ de toxicidade do esquema adjuvante, e 1,4 e 5,3 anos de sobrevivência, respectivamente, em imunodeprimidos e competentes. ${ }^{29,34,35}$ Entretanto, há melhora do prognóstico ${ }^{35}$ e redução do número de displasias ${ }^{36}$ com o uso de coquetel de drogas antirretrovirais.

Encontramos uma recidiva num doente estádio II (50\%) com lesão na margem anal e em três (25\%) com tumor do canal anal, nos estádios II, IIIa e IIIb, respectivamente. Observamos também que os doentes nos estádios mais avançados reincidiram mais, porém a pequena casuística impediu a confirmação pela análise estatística. Revisando a literatura, notamos que os tumores da margem anal têm piores resultados, bem como aqueles nos estádios mais avançados, ${ }^{23,27,32}$ à semelhança do que obtivemos.
O uso de RT/QT combinada apresenta, sobre a RT exclusiva, a vantagem da redução das doses médias de radiação devido à ação sensibilizante da quimioterapia, associando-se, porém, à toxicidade não desprezível de $20-50 \%$, em grau geralmente leve, mas podendo chegar a graus letais. ${ }^{25,33}$ Para doentes HIVpositivos, alguns autores referiram que o tratamento deva ser similar ao dos soronegativos, ${ }^{30,31}$ entretanto, há os que citaram que os efeitos tóxicos agudos e crônicos são mais frequientes nos primeiros, havendo necessidade de ajuste das doses. ${ }^{37}$ Não constatamos óbito na vigência da RT/QT, entretanto tivemos complicações em 57,1\% dos casos, incluindo dermatite, retite, estenose, incontinência anal e fístula retovaginal. Ambos os portadores do HIV apresentaram dermatite de pequena intensidade.

As duas indicações de AAP neste estudo condisseram com as adotadas em outros serviços. São elas os tumores em estádio mais avançado, sem remissão com RT/QT, ou aqueles com recidiva. $^{21,26,37}$

O estádio clínico inicial foi o principal fator prognóstico para sobrevivência global nos trabalhos analisados, ${ }^{25,33}$ fato não observado provavelmente pela nossa casuística pequena. Protocolos utilizando RT/ QT apresentaram taxas de controle local do tumor primário entre 50 e $100 \%$, dependendo do tamanho e do estádio do tumor. ${ }^{19,23,28,38}$ Nossa casuística mostrou taxa de $100 \%$ em doentes nos estádios I e II e de $75 \%$ nos estádios IIIa e IIIb.

Quanto ao tamanho do tumor, foi relatada sobrevivência de cinco anos de $96 \%$ para os T1, 87\% para os T2, 79\% para os T3 e 44\% para os T4. ${ }^{28} \mathrm{Já}$, em relação ao estádio, esses índices alcançaram $100 \%$ para o I, $82 \%$ para o II, $74 \%$ para o IIIa e $18 \%$ para o IIIb. ${ }^{26}$ Em nosso estudo, a sobrevivência global de três anos foi de $78,5 \%$, sendo $100 \%$ nos estádios I e II e $25 \%$ nos estádios IIIa e IIIb. A taxa de recidiva local foi de 30,8\%, sendo $20 \%$ nos estádios I e II e 50\% nos estádios mais avançados. A literatura referiu índices entre $12 \mathrm{e}$ $39 \% .^{26,28,33}$

Apesar do grande número de publicações a respeito do carcinoma anal, são poucas as grandes casuísticas. Esse fato dificulta o exato conhecimento de alguns aspectos da doença, como a associação com o HPV e sua real incidência, o esquema terapêutico especial para imunodeprimidos e para os mais idosos, a dose de radioterapia mais efetiva. Trabalhos 
multicêntricos, prospectivos e randomizados precisam ser feitos para dirimir essas dúvidas. De qualquer forma, sugerimos dividir os doentes desse carcinoma conforme a localização do tumor, se da margem ou do canal anal, e entre imunodeprimidos e imunocompetentes para melhor comparação entre os efeitos do tratamento.

\section{CONCLUSÃO}

A análise dos nossos resultados evidenciou que o esquema de tratamento empregado foi efetivo para o controle local e preservação da função esfincteriana do ânus e que, na falha do tratamento radioquimioterápico, a operação de resgate controlou localmente a doença.

SUMMARY: Chemoradiation became the treatment of choice for anal squamous cells carcinoma (SCC). However, in local recurrence or residual disease, local resection or abdominoperineal resection must be performed.

Objective: the aim of this retrospective study was to analyze results of 14 patients treated for anal SCC.

Method: Anal SCC was diagnosed in 17 patients. They were $14(82.3 \%)$ females and three $(17.8 \%)$ males with ages varying from 36 to 78 years old, and 59.1 years-old in mean. Using TNM classification, they were four (23.6\%) class I, six (35.2\%) class II, four (23.6\%) class IIIa and three $(\mathbf{1 7 . 6 \%})$ class IIIb. All patients were submitted prior to chemoradiation, except for one, in whom local resection was performed. We considered that negative biopsy, performed from 12 to 16 weeks after treatment, would define local control for disease.

Results: Three patients (17.6\%) were lost in the follow-up. So, $14(82.3 \%)$ were accompanied from one up to five years. All patients in I and II TNM class and three in IIIa and IIIb $(\mathbf{7 5 \%})$ had complete tumor remission. We have performed anorectal amputation in two patients and local resection in other two patients. Local recurrence occurred in two I and II TNM class patients (20\%) and in two III and IIIb TNM class patients $(\mathbf{5 0} \%)$. All patients in I and II class were alive after the third year, despite tumor control being obtained in eight patients. In IIIa and IIIb patients, survival after one year was seen in three (75\%) and after the third year in just one $(25 \%)$ patients that remain free from disease. No death occurred during chemoradiation. Two HIV-positive patients presented anal human papillomavirus infection.

Conclusion: Our results showed that the treatment used was effective for local control, preserving anal function, and whenever chemoradiation fails, surgical treatment was effective to control this kind of cancer.

Key words: Anal carcinoma; Chemoradiation; Squamous cells carcinoma.

\section{REFERÊNCIAS BIBLIOGRÁFICAS}

1. Stearns MW Jr, Urmacher C, Sternberg SS, Woodruff J, Attiyeh F. Cancer of the anal canal. Curr Probl Cancer 1980;4:1-44.

2. Ryan DP, Campton CC, Mayer RJ. Carcinoma of the anal canal. N Engl J Med 2000;342:798-800.

3. Moscicki AB, Hills NK, Shiboski S, Darragh TM, Jay N, Powell K et al Risk factors for abnormal anal cytology in young heterosexual women. Cancer Epidemiol Biomarkers Prev 1999; 8:173-8.

4. Modesto VL, Gottesman L. Doenças sexualmente transmitidas e manifestações anais da AIDS. Clin Cir Am Norte 1994;74:1501-34.

5. Lytwyn A, Salit IE, Raboud J, Chapman W, Darragh T, Winkler $B$ et al. Interobserver agreement in the interpretation of anal intraepithelial neoplasia. Cancer.2005;103: 1447-56.

6. Frisch M, Fenger C, van den Brule AJ, Sorensen P, Meijer CJ, Walboomers JM et al. Variants of squamous cell carci- noma of the anal canal and perianal skin and their relation to human papillomaviruses. Cancer Res 1999;59:753-757.

7. Gervaz P, Allal AS, Villiger P, Buhler L, Morel P. Squamous cell carcinoma of the anus: another sexually transmitted disease. Swiss Med Wkly 2003;133:353-9.

8. Berretta M, Cinelli R, Martellotta F, Spina M, Vaccher E, Tirelli U. Therapeutic approaches to AIDS-related malignancies. Oncogene. 2003;22:6646-59.

9. Berry JM, Palefsky JM, Welton ML. Anal cancer and its precursors in HIV-positive patients: perspectives and management. Surg Oncol Clin N Am. 2004;13:355-73.

10. Diamond C, Taylor TH, Aboumrad T, Bringman D, AntonCulver H. Increased incidence of squamous cell anal cancer among men with AIDS in the era of highly active antiretroviral therapy. Sex Transm Dis. 2005;32:314-20.

11. von Krogh G, Wikstrom A, Syrjanen K, Syrjanen S. Anal and penile condylomas in HIV-negative and HIV-positive men: clinical, histological and virological characteristics correlated to therapeutic outcome. Acta Derm Venereol, 1995; 75:470-4. 
12. Aynaud O, Piron D, Barrasso R, Poveda JD. Comparison of clinical, histological, and virological symptoms of HPV in HIV-1 infected men and immunocompetent subjects. Sex Transm Infect. 1998;74:32-4

13. Zanier K, Charbonnier S, Baltzinger M, Nomine Y, Altschuh D, Trave G. Kinetic Analysis of the Interactions of Human Papillomavirus E6 Oncoproteins with the Ubiquitin Ligase E6AP Using Surface Plasmon Resonance. J Mol Biol 2005; 349:401-12.

14. Crook T, Vousden K. Properties of p53 mutations detected in primary and secondary cervical cancers suggest mechanisms of metastasis and involvement of environmental carcinogens. EMBO J 1992;11:3935-3940.

15. Ho L, Chan SY, Chow V, Chong T, Tay SK, Villa LL et al. Sequence variants of Human papillomavirus type 16 in clinical samples permit verification and extension of epidemiological studies and construction of a phylogenetic tree. J Clin Microbiol 1991;29:1765-1772.

16. Matheus CO, Koch KS, Névola AC, Silva JH. Carcinoma epidermóide de canal anal: resultado do tratamento de 46 pacientes. Rev Bras Coloproct 1997; 17:180-5.

17. Khatri VP, Chopra S. Clinical presentation, imaging, and staging of anal cancer. Surg Oncol Clin N Am. 2004;13:295-308.

18. Tarantino D, Bernstein MA. Endoanal Ultrasound in the Staging and Management of Squamous-Cell Carcinoma of the Anal Canal Potential Implications of a New Ultrasound Staging System. Dis Colon Rectum 2002; 45:16-22.

19. Bonnen M, Crane C, Vauthey JN, Skibber J, Delclos ME, Rodriguez-Bigas $\mathrm{M}$ et al. Long-term results using local excision after preoperative chemoradiation among selected $\mathrm{T} 3$ rectal cancer patients. Int J Radiat Oncol Biol Phys 2004;60:1098-105.

20. Nilsson PJ, Svensson C, Goldman S, Glimelius B. Salvage abdominoperineal resection in anal epidermoid cancer. British Journal of Surgery 2002; 89:1425-9.

21. Rodrigues MRS, Magi JC, Corrêa RS, Guerra GR, Souza FS, Fonseca MM et al. Cirurgia de resgate no carcinoma de canal anal. Rev Bras Coloproct 2004;24:137-39.

22. Esiashvili N, Landry J, Matthews RH. Carcinoma of the anus: strategies in management. Oncologist. 2002;7:188-99.

23. Eng C, Abbruzzese J, Minsky BD. Chemotherapy and radiation of anal canal cancer: the first approach. Surg Oncol Clin N Am. 2004;13:309-20, viii.

24. Nigro ND, Vaitkevicius VK, Considine B Jr. Combined therapy for cancer of anal canal: a preliminary report. Dis Colon Rectum 1974; 17:354-6

25. Chauveinc L, Buthaud X, Falcou MC, Mosseri V, De la Rochefordiere A, Pierga JY et al. Anal canal cancer treatment: practical limitations of routine prescription of concurrent chemotherapy and radiotherapy. Br J Cancer 2003;89:2057-61.

26. Ferrigno R, Nakamura RA, Dos Santos Novaes PE, Pellizzon AC, Maia MA, Fogarolli RC et al. Radiochemotherapy in the conservative treatment of anal canal carcinoma: retrospective analysis of results and radiation dose effectiveness. Int J Radiat Oncol Biol Phys. 2005;61:1136-42.

27. Skibber J, Rodriguez-Bigas MA, Gordon PH. Surgical considerations in anal cancer. Surg Oncol Clin N Am. 2004;13:321-38.

28. Deniaud-Alexandre E, Touboul E, Tiret E, Sezeur A, Houry $\mathrm{S}$, Gallot D et al. Results of definitive irradiation in a series of 305 epidermoid carcinomas of the anal canal. Int J Radiat Oncol Biol Phys 2003;56:1259-73.

29. Place RJ, Gregorcyk SG, Huber PJ, Simmang CL. Outcome analysis of HIV-positive patients with anal squamous cell carcinoma. Dis Colon Rectum 2001; 44:506-512.

30. Cleator S, Fife K, Nelson M, Gazzard B, Phillips R, Bower $\mathrm{M}$. Treatment of HIV-associated invasive anal cancer with combined chemoradiation. Eur J Cancer 2000; 36:754-8.

31. Lim ST, Levine AM. Non-AIDS-Defining Cancers and HIV Infection. Curr Infect Dis Rep. 2005;7:227-234.

32. Grabenbauer GG, Kessler H, Matzel KE, Sauer R, Hohenberger W, Schneider IH. Tumor site predicts outcome after radiochemotherapy in squamous-cell carcinoma of the anal region: long-term results of 101 patients. Dis Colon Rectum. 2005;48:1742-51.

33. Renehan AG, Saunders MP, Schofield PF, O'Dwyer ST. Patterns of local disease failure and outcome after salvage surgery in patients with anal cancer. Br J Surg. 2005 ;92:605-14.

34. Sobhani I, Vuagnat A, Walker F, Vissuzaine C, Mirin B, Hervatin F et al. Prevalence of high-grade dysplasia and cancer in the anal canal in human papillomavirus-infected individuals. Gastroenterology 2001;120:857-66.

35. Vatra B, Sobhani I, Aparicio T, Girard PM, Puy Montbrun $\mathrm{TD}$, Housset $\mathrm{M}$ et al. Anal canal squamous-cell carcinomas in HIV positive patients: clinical features, treatments and prognosis. Gastroenterol Clin Biol 2002; 26:150-6.

36. Martin F, Bower M. Anal intraepithelial neoplasia in HIV positive people. Sex Transm Infect 2001;77:327-31.

37. Klencke BJ, Palefsky JM. Anal cancer: an HIV-associated cancer. Hematol Oncol Clin North Am. 2003;17:859-72.

38. Graf R, Wust P, Hildebrandt B, Gogler H, Ullrich R, Herrmann $\mathrm{R}$ et al. Impact of overall treatment time on local control of anal cancer treated with radiochemotherapy. Oncology $2003 ; 65: 14-22$.

\section{Endereço para correspondência:}

\section{LUIS AUGUSTO PALMA DALLAN}

Rua Inhambu, 902 - ap.61-B - Bairro Moema

04.520-013 - São Paulo (SP)

E-mail:luisdallan@yahoo.com 